\title{
Flujo compartido en actividades colectivas físico-deportivas y bienestar subjetivo
}

\author{
Larraitz N. Zumeta, Nekane Basabe, Saioa Telletxea, Alberto Amutio \\ y Magdalena Bobowik \\ Universidad del País Vasco (España)
}

\begin{abstract}
Esta investigación estudia las actividades físico-deportivas como una fuente de flujo compartido o de experiencia óptima y su relación con el bienestar subjetivo. Se presentan dos estudios con estudiantes universitarios $(N=88, N=285)$ que participan en actividades físicas y deportivas en grupo. Las medidas incluyen la calidad de la participación en la actividad, el flujo individual y compartido, las emociones positivas, y el bienestar subjetivo. El primer estudio muestra que el flujo compartido fue más intenso que el individual. En el segundo estudio, se realizaron análisis factoriales confirmatorios $(A F C)$ y análisis de mediación múltiple. Los resultados mostraron una estructura factorial de segundo orden con nueve dimensiones de primer orden, además de los efectos mediadores del flujo compartido y de las emociones positivas en el bienestar subjetivo. Se confirma la relevancia del flujo compartido durante los encuentros colectivos y su relación con el bienestar subjetivo.
\end{abstract}

Palabras clave: Deporte y actividad física, bienestar subjetivo, flujo compartido, emociones compartidas.

Shared flow in collective physical and sports activities and subjective well-being. This study examines physical and sport activities as a source of shared flow or optimal shared experiences and their relationship with subjective well-being (SWB). Two studies are presented with university students $(N=88$ and $N=285)$ participating in group sports and physical activities. Measures included the degree of engagement in the activity, individual and shared flow, positive emotions, and subjective well-being (SWB). The first study showed that shared flow was more intense than individual flow. In the second study, confirmatory factor analyses and multiple mediation analyses were performed. The results showed a second order factorial structure with nine primary dimensions, in addition to mediating effects of shared flow and positive emotions on SWB. The results confirm the relevance of shared flow during collective gatherings and its relations with SWB.

Keywords: Sports and physical activity, subjective well-being, shared flow, shared emotions. 
Existe un creciente interés por el estudio de las experiencias de flujo desde una perspectiva colectiva (Delle Fave, Massimini, y Bassi, 2013; Salanova, Rodríguez-Sánchez, Schaufeli, y Cifre, 2014; Walker, 2010; Zumeta, Oriol, Telletxea, Amutio, y Basabe, 2016). Este estudio plantea analizar el flujo como experiencia compartida en las actividades físicas-deportivas que se realizan en compañía de otras personas.

El flujo se entiende como un estado psicológico óptimo en el que las personas consiguen abstraerse completamente en la ejecución de la tarea, alcanzando un nivel de rendimiento óptimo en el que la actividad se desempeña de forma automática (Jackson y Csikszentmihalyi, 2002). El estado de "flow" puede variar en intensidad y/o profundidad. Un estudio transcultural confirmó que los componentes teóricos del flujo se desarrollan tanto en las experiencias cotidianas, como en las actividades de tiempo libre y deportivas, obteniendo puntuaciones altas (medias mayores de 6 en un rango de 0 a 9) en todos los componentes del flujo salvo en la pérdida de autoconciencia (Delle Fave et al., 2013).

Jackson y Csikszentmihalyi (2002) plantean nueve dimensiones en la experiencia de flujo: (1) equilibrio entre las habilidades personales y el reto que impone la actividad; (2) fusión de acción y conciencia, la persona actúa de manera automática; (3) metas claras; (4) retroalimentación inmediata; (5) concentración enfocada en la actividad, absorción completa; (6) sensación de control sobre la actividad y las propias acciones; (7) pérdida de conciencia del yo, sensación de fusión; (8) distorsión en la percepción temporal; y (9) experiencia intrínsecamente gratificante, o autotélica. Diversos estudios han confirmado la validez de estas nueve dimensiones, así como su asociación con un alto nivel rendimiento y con el afecto positivo (Delle Fave et al., 2013; García-Calvo, Jiménez, Santos-Rosa, Reina, y Cervelló, 2008), aunque la distorsión temporal no se produce en todas las experiencias de flujo.

Tanto las medidas de flujo como estado (intensidad del flujo enfocada en una actividad específica), o como rasgo (experiencia general en distintas situaciones y momentos) obtienen buenas propiedades psicométricas (Moneta, 2012). La validez de constructo estimada a través de modelos factoriales confirmatorios ha sido estudiada con dos propuestas alternativas. La primera postula una estructura de nueve factores correlacionados entre sí, presentando el flujo con un constructo multifactorial. Ésta es la aproximación que se asume en este estudio. En contraposición, se propone un modelo de un único factor que aglutina a todos los ítems. Sin embargo, el modelo de nueve factores arrojó mejores índices de ajuste (Jackson y Eklund, 2002, 2004; Moneta, 2012).

Este estudio pretende dar un enfoque social al estudio de las experiencias óptimas en el deporte. Los estudios de Walker (2010) proporcionan evidencias de que el flujo experimentado en una situación social suscita emociones positivas en un nivel más alto que el flujo solitario. En esta línea, Mesurado (2009) apuntaba que el 60\% de las actividades que generaron un estado óptimo se produjeron en presencia de otros. 
Los estudios basados en la perspectiva de Durkheim (1912) destacan que la participación en actividades colectivas genera emociones compartidas o efervescencia colectiva (Paez, Rimé, Basabe, Wlodarczyk, y Zumeta, 2015). En la misma línea para Csíkszentmihalyi (1990) la participación en rituales colectivos puede concebirse como la oportunidad que la sociedad ofrece a sus miembros para vivir experiencias de flujo de maneras socialmente aceptables.

Estas actividades sociales o colectivas reúnen las características propias de la experiencia de flujo, implican objetivos claros, permiten ajustar el nivel de rendimiento de las propias capacidades, facilitan información clara sobre la calidad de la ejecución, eliminan distracciones y hacen posibles altos niveles de concentración. Asimismo, como han revelado los estudios con deportistas, la sincronía en los movimientos (expresiones faciales, vocalizaciones, posturas y movimientos de una persona a otra) y el foco de atención compartido en la actividad favorece el contagio emocional y genera un buen desempeño. Los deportes en equipo proporcionan oportunidades para desarrollar la motivación de afiliación. Para los atletas de élite jugar eficientemente en equipo significa confiar en los jugadores, compartir sentimientos positivos, sincronizar los movimientos y centrar la atención en los pases, y es entonces, cuando se experimenta flujo (Schiepe-Tiska y Engeser, 2012). Además, la práctica de actividad física produce una multitud de efectos psicológicos positivos (e.g., aumento de la autoconfianza y la autoeficacia, mejora del funcionamiento cognitivo, mayor sensación de bienestar y mejora de los estados emocionales, así como una reducción de la ansiedad, la depresión y el estrés (Brown, Mishra, Lee, y Bauman, 2000; García, Bohórquez, y Fernández, 2014; Sampedro, 2015).

Csikszentmihalyi y Csikszentmihalyi (1998) demostraron que la actividad físico-deportiva en adolescentes es una de las actividades en las que con mayor frecuencia se experimenta flujo. El estudio transcultural anteriormente citado de Delle Fave et al. (2013) mostró que las experiencias óptimas (flujo) son comunes y universales: el 85\% de las personas reportaron flujo en una o más de las actividades de su vida cotidiana, de estas personas el $54 \%$ eligió actividades estructuradas como deportes y actividad física (52\%) o aficiones y juegos $(47 \%)$.

En base a lo expuesto se postula que en las actividades colectivas físico-deportivas se producen, por un lado, emociones colectivas como la "efervescencia emocional" (Fredickson, 2009), que se expresan y se comparten públicamente y, al mismo tiempo, una "experiencia de flujo" compartida con los otros, como estado de motivación intrínseca en el que las personas se implican en la acción por los efectos psicológicos positivos que conlleva (Csikszentmihanyi, 1990).

Sin embargo, la relación entre flujo y felicidad no es automática; ambos están correlacionados, pero no son equivalentes. Según Engeser (2012), cuando una persona está inmersa en una actividad no tiene la conciencia de ser feliz, dado que la experiencia 
de fluir está más relacionada con el interés y la activación positiva que con la felicidad, siendo ésta última una consecuencia más del flujo. Al mismo tiempo, la calidad de la participación en la actividad o la relación entre las habilidades y el reto que la actividad supone influyen en el afecto positivo mediante el flujo experimentado (efecto de mediación) (Landhäuber y Keller, 2012).

Desde esta perspectiva se plantea que la participación en actividades deportivas colectivas o grupales serán una fuente de experiencias óptimas que se intensifican emocionalmente por ser compartidas, simultáneas y coordinadas, y que, en general, son más placenteras al provocar más emociones positivas que las experiencias óptimas individuales (Walker, 2010).

Por lo tanto, el objetivo general de esta investigación es constatar las diferencias existentes entre la experiencia individual y compartida del flujo y mostrar que el flujo en actividades físico-deportivas colectivas produce experiencias óptimas compartidas, altas emociones positivas y efectos positivos sobre la experiencia de bienestar.

\section{ESTUDIO 1}

El objetivo de este primer estudio es comprobar la existencia de diferencias en la percepción del flujo compartido con respecto a la percepción del flujo disposicional individual experimentada durante la participación en una actividad colectiva físico-deportiva. Asimismo, analizar las diferencias en la experiencia de flujo en función de la calidad de la participación (baja-alta) y el tipo de actividad físico-deportiva. Se espera que la experiencia de flujo compartido sea más intensa que la experiencia individual $(H 1)$. Así mismo, se postula que, a mayor calidad de participación, más intensa será la experiencia de flujo durante la actividad físico-deportiva (H2).

\section{MÉTODO}

\section{Participantes}

La muestra estuvo compuesta por 88 estudiantes universitarios voluntarios y voluntarias (76\% mujeres) de entre 19 y 36 años $(M=20.69, D T=2.56)$. Todos los participantes practicaban algún tipo de actividad colectiva físico-deportiva cada semana. Las actividades reportadas fueron las siguientes: (1) $34.10 \%$ actividades deportivas colectivas federadas y no federadas (e.g., football, basketball, etc.), (2) $23.90 \%$ actividades físicas o deportivas en grupo (e.g., aerobic, bicicleta estática, etc.), y (3) 42\% actividades físicas recreativas con otras personas (e.g., actividades al aire libre: senderismo, correr, etc.). 


\section{Instrumentos}

Calidad de la participación en la actividad. Se realizaron cinco preguntas ad hoc en relación a la actividad: importancia, intensidad, satisfacción, implicación y orgullo (rango de respuesta: $1=$ nada en absoluto, $7=$ mucho), y se calculó la puntuación media. El alpha Cronbach $(\alpha)$ de la escala fue de .90 .

Escala de Flujo Disposicional (EFD). Versión reducida de la Dispositional Flow Scale (Jackson y Marsh, 1996) en versión española (García-Calvo, Jiménez, SantosRosa, Reina, y Cervelló, 2008). Se compone de 27 ítems, 3 por dimensión. El formato de respuesta oscilaba de 1 (nunca) a 7 (siempre). El alpha de Cronbach $(\alpha)$ de la escala fue de .94 .

Escala de Flujo Compartido (EFC). Formulada para explorar el flujo compartido en las actividades colectivas físico-deportivas (Zumeta, Oriol, Telletxea, Amutio, y Basabe, 2016) a partir de la versión española de Garcia et al. (2008) (ver tabla 3). El rango de respuesta fue de 1 (nunca) a 7 (siempre). El $\alpha$ para la escala total fue .95 .

\section{Procedimiento}

Se administró una encuesta online a los estudiantes universitarios que participaron voluntariamente en el estudio. Previamente se les proporcionó la información respecto a la investigación y firmaron el consentimiento informado. Los datos fueron codificados de forma alfanumérica, asegurando el anonimato y el cumplimiento de la Ley de Protección de Datos Personales del Comité de Ética de Investigación. Los participantes debían especificar el tipo de actividad física con otras personas en la que habían participado la semana anterior y completar la versión individual y compartida de la escala de flujo.

\section{Análisis}

Se calcularon las diferencias de medias (t-test) entre las puntuaciones EFD y las EFC, y se utilizó la $d$ de Cohen para calcular el tamaño del efecto (TE) (Cohen, 1988), con el programa $\mathrm{G} *$ Power 3.1. Además, se realizó un análisis de varianza (ANOVA) en función de la calidad de la participación alto o baja (según la mediana). Se utilizó el programa SPSS 21.0.

\section{RESULTADOS}

En la tabla 1 se muestran los estadísticos descriptivos $(M, D T$ y valores $t)$ de las medidas de EFD y de la EFC. Todas las puntuaciones medias son superiores al punto medio de la escala ( $>4.5$, en un rango de respuesta de 1 a 7 ). Con respecto a los valores medios en las dimensiones, las mayores puntuaciones se obtuvieron en las dimensiones de experiencia autotélica y metas claras para ambas escalas. Por su parte, las puntuaciones 
más bajas correspondieron a las dimensiones de distorsión temporal y pérdida de autoconciencia.

Con un tamaño muestral de 88 personas, se observaron diferencias con una magnitud de tamaño del efecto pequeña (TE<.27) (Cohen, 1988). Así y todo, las puntuaciones globales en flujo compartido fueron superiores a las puntuaciones globales en flujo individual $(H 1)$. Igualmente, en ninguna de las dimensiones las puntuaciones EFD superaron a las puntuaciones de EFC. Sobresalen las puntuaciones en las dimensiones: equilibrio habilidad-reto, metas claras y retroalimentación inmediata (TE>.25), con diferencias significativas entre EFD y EFC (véase tabla 1). No hubo diferencias significativas en fusión acción-conciencia, concentración en la tarea, sensación de control y experiencia autotélica.

Tabla 1. Descriptivos y diferencias de medias (t-test) entre flujo individual (EFD) y flujo compartido (EFC)

\begin{tabular}{lccccc}
\hline \multirow{2}{*}{ Variables } & EFD & EFC & \multirow{2}{*}{$t_{(87)}$} & $p$ & \multirow{2}{*}{$d$} \\
\cline { 2 - 4 } & $M(D T)$ & $M(D T)$ & & \\
\hline Habilidad-reto & $5.37(1.01)$ & $5.57(1.04)$ & -2.53 & .013 & 0.269 \\
\hline Fusión acción-conciencia & $5.40(0.97)$ & $5.35(1.00)$ & 0.60 & .554 & 0.063 \\
\hline Metas claras & $5.69(1.05)$ & $5.83(1.10)$ & -2.37 & .020 & 0.253 \\
\hline Retroalimentación inmediata & $5.14(1.13)$ & $5.32(1.00)$ & -2.45 & .016 & 0.261 \\
\hline Concentración en la tarea & $5.41(1.16)$ & $5.37(1.10)$ & 0.51 & .608 & 0.055 \\
\hline Sensación de control & $5.29(1.08)$ & $5.40(0.98)$ & -1.34 & .183 & 0.143 \\
\hline Perdida de auto-conciencia & $4.65(1.83)$ & $4.87(1.92)$ & -1.93 & .057 & 0.206 \\
\hline Distorsión del tiempo & $4.51(1.46)$ & $4.69(1.53)$ & -1.99 & .050 & 0.212 \\
\hline Experiencia autotélica & $5.83(0.95)$ & $5.94(0.95)$ & -1.54 & .127 & 0.164 \\
\hline Total Flujo & $5.28(0.85)$ & $5.38(0.85)$ & -2.31 & .023 & 0.246 \\
\hline $\begin{array}{l}\text { Nota: } N=88 ; \text { EFD=escala de flujo disposicional individual, EFC=escala de flujo compartido; } d=\mathrm{d} \text { de } \\
\text { Cohen. }\end{array}$ & & & & &
\end{tabular}

En lo que respecta al tipo de actividades físico-deportivas, no se obtuvieron diferencias estadísticamente significativas en las puntuaciones de EFD y de EFC $\left(\mathrm{p}_{\mathrm{s}}>\right.$.05). Sin embargo, para actividades deportivas colectivas (federadas y no federadas) se obtuvo una diferencia de medias significativa en la dimensión de metas claras a favor del flujo compartido en comparación con el individual $\left(M_{\mathrm{EFD}}=6.09, D T=0.71\right.$; $\left.M_{\mathrm{EFC}}=6.28, D T=0.79 ; t_{(29)}=2.036, p=.051, d=0.370\right)$.

En la tabla 2 se muestran las diferencias en cada escala de las dimensiones del flujo con respecto a la alta y baja calidad de participación. En todos los casos se encontraron diferencias significativas, excepto en la dimensión de distorsión temporal del EFD, de modo que cuando la calidad de participación es alta ambos tipos de flujo son más intensos (H2).

Además, las pruebas t-test entre EFD y EFC para baja y alta calidad de participación por separado, revelaron que cuando la calidad de la participación es alta, las puntuaciones globales son mayores para EFC que para EFD $\left(t_{(44)}=-1.98, p=.054\right.$, $d=0.294$ ). Igualmente, al comparar las puntuaciones medias por dimensión entre las escalas en función de la calidad de la participación, EFC es especialmente más intenso en 
las dimensiones de habilidad-reto $\left(t_{(44)}=-2.53, \quad p=.015, d=0.378\right)$ y metas claras $\left(t_{(44)}=-2.04, p=.047, d=0.310\right)$. Finalmente, con baja calidad de participación solo se encontraron diferencias en la retroalimentación inmediata $\left(t_{(42)}=-2.19, \quad p=.034\right.$, $d=0.334$ ), mayor en la EFC que en la EFD.

Tabla 2. Contraste de medias (ANOVA) entre EFD y EFC en función de la calidad de participación (baja $\mathrm{CP} /$ alta $\mathrm{CP}$ )

\begin{tabular}{|c|c|c|c|c|c|c|c|c|c|c|}
\hline \multirow[b]{2}{*}{ Variables } & \multicolumn{2}{|c|}{ EFD } & \multirow[b]{2}{*}{$F_{(1,86)}$} & \multirow[b]{2}{*}{$p$} & \multirow[b]{2}{*}{$\eta_{p}^{2}$} & \multicolumn{2}{|c|}{ EFC } & \multirow[b]{2}{*}{$F_{(1,86)}$} & \multirow[b]{2}{*}{$p$} & \multirow[b]{2}{*}{$\eta_{p}^{2}$} \\
\hline & $\begin{array}{l}\text { baja CP } \\
M(D T) \\
\end{array}$ & $\begin{array}{l}\text { alta CP } \\
M(D T) \\
\end{array}$ & & & & $\begin{array}{l}\text { baja CP } \\
M(D T) \\
\end{array}$ & $\begin{array}{l}\text { alta CP } \\
M(D T) \\
\end{array}$ & & & \\
\hline Habilidad-reto & $4.99(0.87)$ & $5.73(1.00)$ & 13.62 & $<.001$ & 0.137 & $5.12(1.12)$ & $6.00(0.74)$ & 18.82 & $<.001$ & 0.180 \\
\hline Fusión acción-conciencia & $5.09(0.86)$ & $5.69(0.98)$ & 9.14 & .003 & 0.096 & $4.96(1.05)$ & $5.73(0.79)$ & 14.97 & $<.001$ & 0.148 \\
\hline Metas claras & $5.31(1.08)$ & $6.06(0.88)$ & 12.71 & $<.001$ & 0.129 & $5.44(1.20)$ & $6.20(0.87)$ & 11.62 & .001 & 0.119 \\
\hline Retroalimentación inmediata & $4.71(1.14)$ & $5.54(0.97)$ & 13.56 & $<.001$ & 0.136 & $4.94(1.13)$ & $5.69(0.70)$ & 14.25 & $<.001$ & 0.142 \\
\hline Concentración en la tarea & $4.80(1.09)$ & $6.00(0.89)$ & 32.07 & $<.001$ & 0.272 & $4.88(1.03)$ & $5.84(0.97)$ & 19.96 & $<.001$ & 0.188 \\
\hline Sensación de control & $4.92(1.00)$ & $5.64(1.04)$ & 11.00 & .001 & 0.133 & $4.97(0.97)$ & $5.81(0.81)$ & 19.59 & $<.001$ & 0.186 \\
\hline Perdida de auto-conciencia & $4.25(1.53)$ & $5.04(2.02)$ & 4.32 & .041 & 0.048 & $4.39(1.83)$ & $5.33(1.91)$ & 5.54 & .021 & 0.061 \\
\hline Distorsión del tiempo & $4.22(1.38)$ & $4.80(1.48)$ & 3.63 & .060 & 0.040 & $4.35(1.41)$ & $5.01(1.59)$ & 4.30 & .041 & 0.048 \\
\hline Experiencia autotélica & $5.38(0.87)$ & $6.27(0.83)$ & 23.97 & $<.001$ & 0.218 & $5.56(0.97)$ & $6.31(0.77)$ & 16.24 & $<.001$ & 0.159 \\
\hline Total Flujo & $4.87(0.73)$ & $5.66(0.77)$ & 24.26 & $<.001$ & 0.220 & $4.97(0.83)$ & $5.77(0.67)$ & 24.98 & $<.001$ & 0.225 \\
\hline
\end{tabular}

Nota: $\mathrm{EFD}=$ escala de flujo disposicional individual, $\mathrm{EFC}=$ escala de flujo compartido; baja $\mathrm{CP}=$ baja calidad de participación en la actividad ( $N=43)$, alta $\mathrm{CP}=$ alta calidad de participación en la actividad $(N=45)$.

\section{CONCLUSIONES}

Los resultados de este estudio señalan que la práctica de actividades físicodeportivas en compañía de otras personas es una fuente sustancial para fluir, tanto de manera individual como compartida. Una condición importante para vivir estas experiencias es que las metas de la actividad sean claras y accesibles y se caractericen por ser experiencias autotélicas, es decir, un fin en sí mismas. Menos importante es el papel de otros aspectos como la distorsión temporal y la pérdida de auto-conciencia.

Se ha podido acreditar la existencia de diferencias entre la experiencia individual de flujo y la experiencia compartida, siendo esta última más fuerte. A pesar de los aspectos comunes de las percepciones de la experiencia de flujo (fusión acciónconciencia, concentración, sensación de control y experiencia autotélica), es cierto que la experiencia compartida de fluir ha destacado en algunas facetas, reportando mayor intensidad en la percepción del equilibrio entre las habilidades y el reto, las metas se perciben más claras y más alcanzables, y provee mayor información sobre el desempeño durante la actividad. De esta manera, se confirma la hipótesis de que en las actividades 
colectivas físico-deportivas la experiencia compartida de flujo es más intensa que la individual, siendo estas diferencias más marcadas cuando la calidad de participación en la actividad es alta.

La calidad de la participación ha mostrado ser importante en la potencia de la experiencia de flujo, tanto a nivel individual como compartido y ha arrojado diferencias en todos los aspectos de la experiencia de fluir entre baja y alta calidad de participación. Así, tanto a nivel global como en las dimensiones del fluir (equilibrio habilidad-reto y metas claras) resulta más relevante la percepción compartida cuanto mayor es la calidad de participación en la actividad. También, cuando la implicación en la actividad es baja, la experiencia compartida provee más retroalimentación sobre el desempeño en la actividad colectiva físico-deportiva.

\section{ESTUDIO 2}

Se postula que el flujo compartido es un constructo único (Delle Fave et al., 2013; García-Calvo et al., 2008; Jackson y Csikszentmihalyi, 2002), que se configura según un modelo factorial de $2^{\circ}$ orden con 9 dimensiones ( $1^{\circ}$ orden) (Jackson y Eklund, 2002, 2004; Moneta, 2012) (H3). Además, dado que la participación en actividades colectivas genera emociones positivas (Páez et al., 2015) y experiencia de flujo compartido, se espera encontrar un efecto mediador del flujo y de las emociones compartidas sobre el bienestar subjetivo (Landhäuber y Keller, 2012) (H4).

\section{MÉTODO}

\section{Participantes}

La muestra estuvo compuesta por 285 estudiantes universitarios voluntarios (71.5\% mujeres) de entre 19 y 30 años $(M=21.62, D T=1.83)$. Las personas participantes practicaron actividades colectivas deportivas federadas y no federadas (35\%), actividades físicas o deportivas en grupo (14\%), y actividades físicas recreativas o de tiempo libre con otras personas $(51 \%)$.

\section{Instrumentos}

Calidad de la participación en la actividad (ver estudio 1). El coeficiente $\alpha$ fue .90 .

Escala de Flujo Compartido (EFC) (ver estudio 1). El coeficiente alpha de Cronbach $(\alpha)$ fue 93 .

Emociones Positivas (Positivity Test; Fredrickson, 2009). Evalúa 10 emociones positivas (e.g., diversión, alegría, asombro, gratitud, esperanza, orgullo, inspiración, amor, serenidad, calma). Las respuestas se evalúan desde 0 (nada) a 4 (mucho). El coeficiente alpha de Cronbach $(\alpha)$ fue .83 . 
Índice de Felicidad de Pemberton (PHI). Se utilizó la dimensión positiva de la escala para medir bienestar subjetivo (Vázquez y Hervás, 2013). Contiene 5 ítems (e.g. "He hecho algo que realmente disfruto haciendo") que se evalúan desde 0 (completamente en desacuerdo) a 10 (completamente de acuerdo). El coeficiente alpha de Cronbach $(\alpha)$ fue .73 .

\section{Procedimiento}

Estudiantes universitarios participaron voluntariamente y completaron los cuestionarios en las aulas. Como en el estudio 1 se aplicó el protocolo de la Ley de Protección de Datos.

\section{Análisis de datos}

Se contrasta la validez estructural de la escala de flujo compartido, por medio de Análisis Factorial Confirmatorio (AFC), con estimación máxima verosimilitud (MLE) con el programa Mplus 6.1. Los índices CFI y TLI, permiten comparar modelos, se consideran valores mínimos aceptables alrededor de .90 y un buen ajuste para valores $>.95$ (Byrne, 2011, p. 70). Los índices de falta de ajuste RMSEA deben situarse entre .05 y .08 (Hu y Bentler, 1999). También se aplicaron modelos de mediación múltiple aplicando el macro PROCESS para SPSS con estimación de los efectos indirectos con el método bootstrap (5000 repeticiones) (Preacher y Hayes, 2008).

\section{RESULTADOS}

En la tabla 3 se muestran los 27 ítems y los estadísticos descriptivos de la escala EFC. Todas las puntuaciones medias son superiores al valor 5 (rango de 1 a 7), revelando experiencias de flujo compartido de alta intensidad. Los ítems con mayores medias correspondieron a la faceta equilibrio habilidad-reto (EFC9), metas claras (EFC12 y EFC21) y experiencia autotélica (EFC27). Por el contrario, distorsión temporal (EFC26) y fusión acción-conciencia (EFC11 y EFC20) presentaron las puntuaciones más bajas.

Para contrastar la dimensionalidad de la escala se probaron tres modelos: el Modelo A de un único factor, el Modelo B con una estructura factorial de primer orden compuesta por las nueve dimensiones independientes del flujo, y el modelo $\mathrm{C}$ de segundo orden, con 9 factores de primer orden (ver tabla 4). El modelo $\mathrm{C}$ presenta índices de ajuste sustancialmente mejores que los modelos A y B, y en los límites establecidos (CFI=.916) (H3). 
Tabla 3. Escala Flujo Compartido: ítems y descriptivos

\begin{tabular}{|c|c|c|c|}
\hline & & $M$ & $D T$ \\
\hline$\overline{\mathrm{EFC} 1}$ & Sabíamos que nuestras capacidades nos permitían hacer frente al desafío que se nos planteaba. & 5.33 & 1.33 \\
\hline EFC2 & Hacíamos las cosas espontánea y automáticamente. & 5.16 & 1.39 \\
\hline EFC3 & Nuestros objetivos estaban claramente definidos. & 5.25 & 1.48 \\
\hline EFC4 & Hemos tenido realmente claro que lo estábamos haciendo bien. & 5.19 & 1.31 \\
\hline EFC5 & Nuestra concentración estaba dirigida a lo que estábamos haciendo. & 5.46 & 1.33 \\
\hline EFC6 & Hemos compartido un sentimiento de control total. & 5.00 & 1.30 \\
\hline EFC7 & No nos importaba lo que los demás pudieran pensar de nosotros. & 5.32 & 1.69 \\
\hline EFC8 & Nos parecía que el tiempo pasaba más rápido o más lento. & 5.39 & 1.42 \\
\hline EFC9 & Nos ha gustado lo que estábamos haciendo. & 5.98 & 1.15 \\
\hline$\underline{\mathrm{EFC} 10}$ & Nuestras habilidades estaban al mismo nivel de lo que nos exige la situación. & 5.32 & 1.23 \\
\hline$\underline{E F C 11}$ & Nos parecía que todo estaba sucedido automáticamente & 4.89 & 1.30 \\
\hline$\underline{\mathrm{EFC} 12}$ & Estábamos seguros de lo que queríamos hacer & 5.75 & 1.16 \\
\hline EFC13 & Sabíamos lo bien que lo estábamos haciendo & 5.29 & 1.24 \\
\hline EFC14 & Estábamos totalmente centrados en lo que estábamos haciendo. & 5.51 & 1.35 \\
\hline EFC15 & Hemos sentido que podíamos controlar lo que estábamos haciendo. & 5.39 & 1.26 \\
\hline$\underline{E F C 16}$ & No nos preocupaba lo que otros pudieran estar pensando de nosotros. & 5.30 & 1.76 \\
\hline$\underline{\mathrm{EFC} 17}$ & El paso del tiempo nos ha parecido diferente a lo normal. & 5.19 & 1.49 \\
\hline EFC18 & Todos hemos encontrado la experiencia que hemos tenido juntos muy valiosa y reconfortante. & 5.50 & 1.26 \\
\hline EFC19 & $\begin{array}{l}\text { Hemos sentido que éramos lo suficientemente buenos para hacer frente al reto o dificultad de la } \\
\text { situación. }\end{array}$ & 5.35 & 1.27 \\
\hline$\underline{\mathrm{EFC} 20}$ & Ejecutábamos automáticamente & 4.95 & 1.36 \\
\hline EFC21 & Sabíamos lo que queríamos conseguir. & 5.73 & 1.23 \\
\hline$\underline{\mathrm{EFC} 22}$ & Estábamos seguros de que, en ese momento, lo estábamos haciendo muy bien. & 5.27 & 1.30 \\
\hline EFC23 & Nos hemos sentido totalmente absorbidos por lo que hacíamos. & 5.21 & 1.56 \\
\hline$\overline{\mathrm{EFC} 24}$ & Hemos sentido un control total de nuestros cuerpos & 5.13 & 1.37 \\
\hline$\underline{\mathrm{EFC} 25}$ & No estábamos preocupados por la imagen que dábamos a los demás. & 5.06 & 1.87 \\
\hline EFC26 & Hemos sentido como si el tiempo se parase & 4.30 & 1.81 \\
\hline EFC27 & La experiencia grupal nos ha dejado una buena impresión, un buen sabor de boca. & 5.71 & 1.32 \\
\hline
\end{tabular}

Tabla 4. Escala flujo compartido: Análisis Factorial Confirmatorio e índices de bondad de ajuste

\begin{tabular}{|c|c|c|c|c|c|c|}
\hline & S-B $\chi^{2}$ & $\mathrm{gl}$ & CFI & TLI & RMSEA & $\begin{array}{c}90 \% \text { del IC } \\
\text { RMSEA }\end{array}$ \\
\hline A. Unifactorial & $2329.262 *$ & 324 & .476 & .432 & .149 & $.144, .155$ \\
\hline B. Factorial 1er orden & $1705.719^{*}$ & 324 & .641 & .611 & .124 & $.119, .130$ \\
\hline C. Factorial de $2^{\circ}$ orden & $637.934 *$ & 315 & .916 & .906 & .061 & $.054, .068$ \\
\hline
\end{tabular}

El modelo C de segundo orden permite comprobar que el EFC está fuertemente definido por las dimensiones de equilibrio habilidad-reto, metas claras, retroalimentación inmediata, control, concentración y experiencia autotélica, todas ellas con cargas factoriales altas $(F>$.80). Las dimensiones con menor peso son fusión acción-conciencia, pérdida del yo y distorsión temporal sucesivamente $(F>.53)$ (ver figura 1).

Por último, se analizó el papel mediador del EFC y las emociones positivas sobre el índice de felicidad (H4). El análisis de regresión confirmó un efecto significativo de la calidad de participación sobre el EFC y las emociones positivas. Así mismo, el EFC y las emociones positivas fueron predictores significativos y positivos de la felicidad. 
Figura 1. Escala de Flujo Compartido (EFC): Modelo factorial confirmatorio de $2^{\circ}$ orden

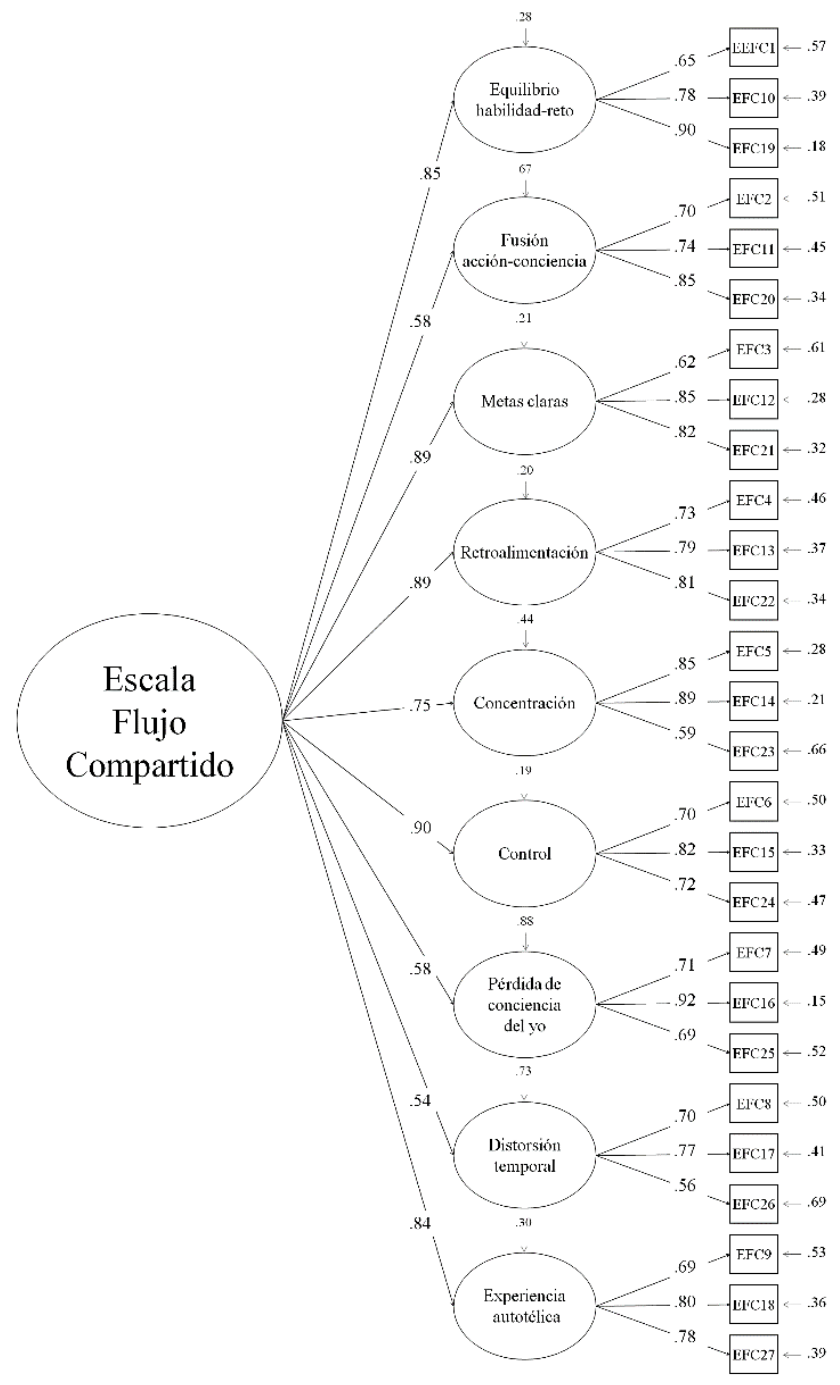

También, mostró una relación significativamente positiva entre ambas variables mediadoras (EFC y emociones positivas). Adicionalmente, se encontró un efecto total significativo de la calidad de la participación en la felicidad $(B=0.11)$. Sin embargo, este efecto dejó de ser significativo, una vez introducidos ambos mediadores en el modelo ( $B=-$ 0.07), lo que señala un efecto de mediación completa (ver figura 2). Hubo un efecto 
indirecto significativo de la calidad de participación en la actividad sobre la felicidad a través de EFC $(B=0.06, E T=.02,95 \%$ CI [0.011, 0.126]), e igualmente a través de las emociones positivas $(B=0.10, E T=.03,95 \%$ CI $[0.049,0.172])$. Además, fue significativo el efecto indirecto secuencial de la calidad de participación a través del EFC, lo que a su vez aumentó las emociones positivas ( $B=0.02, E T=.008,95 \%$ CI $[.004, .038])$.

Figura 2. Calidad de la participación y felicidad: Modelo de mediación múltiple

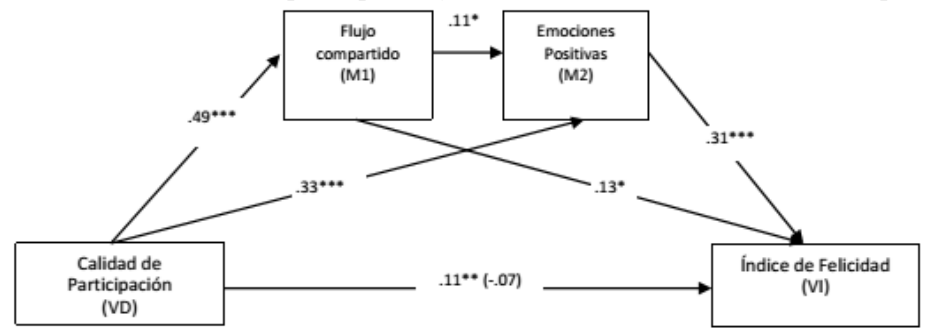

Nota: Coeficientes estandarizados $(* p<.05, * * p<.01, * * * p<.001)$.

\section{CONCLUSIONES}

Este estudio ha contrastado la estructura factorial de flujo en el caso de actividades físico-deportivas, comprobándose que la experiencia de flujo compartido se estructura en las nueve dimensiones teóricas, al igual que sucede con los estudios del flujo individual (García-Calvo et al., 2008). El modelo factorial de segundo orden mostró los mejores índices de ajuste en comparación con los modelos alternativos, congruentemente con otros estudios (Jackson y Eklund, 2002, 2004; Moneta, 2012).

Asimismo, se ha observado que no todos los componentes del flujo adquieren la misma importancia para la experiencia óptima, en concreto, las facetas relativas a la fusión acción-conciencia, pérdida del yo, y distorsión temporal tuvieron menos peso en el constructo. Al mismo tiempo, y confirmando que el estado de flujo puede variar de intensidad, las puntuaciones medias mostraron que la prevalencia de las experiencias de distorsión temporal y fusión acción-conciencia era menor que el resto. Previamente, otros estudios ya habían destacado que, en actividades de tiempo libre y deporte, la pérdida de autoconciencia era la experiencia menos común (Delle Fave et al., 2013).

Se postulaba que la participación en actividades físico-deportivas colectivas promueve experiencias de flujo que se intensifican emocionalmente por ser compartidas, simultáneas y coordinadas (Páez et al., 2015), y que se asociará a una experiencia emocional positiva, mejorando el bienestar (Delle Fave et al., 2013; Landhäuber y Keller, 2012). Los resultados constataron que las actividades colectivas físico-deportivas se asociaron a las experiencias de felicidad, facilitadas por la experiencia de flujo compartido y la efervescencia emocional (emociones colectivas), que se generan y comparten durante 
la actividad en grupo, como han mostrado los efectos directos e indirectos (H4). Es más, los efectos positivos de la calidad de la participación en la actividad colectiva son explicados (mediados) completamente por las variables flujo compartido y emociones positivas.

\section{DISCUSIÓN GENERAL}

Las experiencias de flujo son muy habituales y universales (Delle Fave et al., 2013). Además, es frecuente que se produzcan en compañía de otros (Mesurado, 2009) y entre ellas las actividades colectivas físico-deportivas están entre las más practicadas, produciendo experiencia autotélicas.

Como ya apuntaban los estudios sobre experiencias colectivas (Delle Fave et al., 2013; Walker, 2010; Zumeta et al., 2016), los encuentros colectivos generan una experiencia compartida que se intensifica cuando se comparte una tarea común, que es desafiante pero no supera las habilidades de la persona, con metas claras, lo que permite que se experimenten las distintas manifestaciones del fluir y resulte en una experiencia autotélica. Este estudio ha puesto de manifiesto la importancia de la experiencia colectiva del fluir.

Se ha mostrado que estas manifestaciones son comunes a distintos tipos de actividades físico-deportivas, si bien en los deportes colectivos parece más importante que haya unos objetivos o metas claras que en otro tipo de actividades más lúdicas. Sin embargo, la experiencia de flujo no es homogénea, en consonancia con otras evidencias (Moneta, 2012), la distorsión temporal y la pérdida de auto-conciencia no aparece en todos los casos, o al menos aparecen menos acentuadas que otras facetas del flujo.

Una de las aportaciones más novedosas de esta investigación ha sido analizar el carácter compartido y colectivo de las experiencias óptimas, o de flujo compartido, confluyendo con otros autores que destacan que cuando el flujo se experimenta en situaciones sociales suscita emociones positivas en un grado más alto que el flujo solitario (Walker, 2010). Estimula compartir con otros las emociones, teniendo la oportunidad de experimentar flujo de manera socialmente deseable (Csikszentmihalyi, 1990), y promoviendo el afecto positivo y el bienestar eudaimónico (Páez et al., 2015). Asimismo, la aportación teórica de este trabajo ha consistido en contrastar que las conductas sincronizadas que ocurren en las actividades físico-deportivas, con movimientos, gestos compartidos, al mismo paso y, en general, el hacer cosas juntos, promueven en los participantes afectos positivos. Este último aspecto es especialmente importante cuando la experiencia es intrínsecamente motivante y absorbente, esto es, cuando se promueve la experiencia de fluir con los otros. Sin embargo, las investigaciones actuales destacan que estas experiencias colectivas ocurren cuando las actividades coordinadas resultan significativas y son emocionales (Wiltermuth y Heath, 2009). Es decir, no es simplemente 
el efecto de la sincronización de movimientos, sino que la actividad debe tener un sentido colectivo, como en el caso de los rituales que refuerzan las creencias y valores sociales (Páez et al., 2015). Concretamente, este estudio ha mostrado que la participación en una actividad colectiva que es percibida como importante, intensa, y satisfactoria conduciría a promover la experiencia del fluir con otros, donde destacan las emociones de diversión y alegría, pero también las emociones transcendentes (más allá del propio sujeto) como la gratitud, la esperanza, la inspiración o la serenidad.

Sin embargo, tal y como otros estudios han destacado, no todas las experiencias de flujo necesariamente producen bienestar, y por tanto, la relación entre flujo y felicidad no es automática (Engeser, 2012). Incluso pueden producirse efectos estresantes y negativos en las experiencias con una excesiva implicación en la actividad (Peifer, 2012). No obstante, en este estudio las actividades físico-deportivas durante el tiempo libre y la recreación aparecen vinculadas a las experiencias placenteras y felices. Y esto era más intenso cuando la experiencia de flujo era compartida.

Entre las limitaciones del estudio cabe mencionar su naturaleza transversal que no permite establecer efectos causales, si bien estudios longitudinales (Páez et al., 2015) han mostrado resultados concordantes con los de este estudio. Resulta necesario avanzar en el diseño de estudios longitudinales y experimentales para mostrar los efectos de la participación en actividades físicas. Por supuesto, también el tamaño muestral limita los resultados. Por otro lado, hay algunas cuestiones que deberían ser desarrolladas con más profundidad en futuros estudios, como los efectos diferenciales de los distintos tipos de actividades físico-deportivas, el tipo de grupos implicados, así como el rol diferencial de las distintas facetas del flujo y su papel según el tipo de actividad y grupo deportivo. Con todo, se considera que este estudio puede hacer avanzar el conocimiento sobre la relación entre actividades físicas compartidas como fuente de flujo y felicidad.

\section{Agradecimientos}

Esta investigación ha sido financiada por el MINECO, PSI2014-51923-P, el Gobierno Vasco y la UPV/EHU [IT-666-13; US13/11; PRE_2013_1_738; UFI 11/04].

Expresamos nuestro agradecimiento a los y las estudiantes participantes, así como a Daniela Alves, MD, por su ayuda en el trabajo de campo. In Memorian a Susana Irazusta, PhD (RIP) por su ejemplar cooperación, apoyo y entusiasmo.

\section{REFERENCIAS}

Brown, W., Mishra G., Lee, C., y Bauman A. (2000). Leisure time physical activity in Australian women: relationship with being and symptoms. Research Quarterly for Exercise and Sport, 71(3), 206-216. doi: 10.1080/02701367.2000.10608901

Byrne, B. (2011). Structural Equation Modeling with Mplus. London, Routledge, Taylor y Francis. 
Cohen, J. (1988). Statistical power analysis for behavioral sciences. Hilsdale: Lawrance Erlbaum Associates.

Csikszentmihanyi, M. (1990). Flow. The psychology of optimal experience. Harper y Row. [traducción española: Flow (Fluir). Psicología de la felicidad. Barcelona, Kairós].

Csikszentmihalyi, M., y Csikszentmihalyi, S. (1998). La medición del flujo en la vida cotidiana [Introducción]. En M. Csikszentmihalyi y S. Csikszentmihalyi (Eds.), Experiencia óptima. Estudios psicológicos del flujo en la conciencia (pp. 93-98). Bilbao: Desclée De Brouwer.

Delle Fave, A., Massimini, F., y Bassi, M. (2013). Psychological selection and optimal experience across cultures. New York: Springer.

Durkheim, E. (1912). Les formes élémentaires de la vie religieuse [The Elementary Forms of Religious Life]. Paris: Alcan.

Engeser, S. (2012). Advances in Flow-Research. New York: Springer. doi: 10.1007/978-1-46142359-1

Fredrickson, B. (2009). Positivity. New York: Crown Publishers. doi: 10.1080/17439760903157109

García, A.J., Bohórquez, M.R., y Fernández, M.L. (2014). La implicación en la actividad física como fuente de felicidad en personas mayores. European Journal of Investigation in Health, Psychology and Education, 4(1), 19-30. doi: 10.1989/ejihpe.v4i1.37

García-Calvo, T., Jiménez, R., Santos-Rosa, F.J., Reina, R., y Cervelló, E. (2008). Psychometric Properties of the Spanish version of the Flow State Scale. The Spanish Journal of Psychology, 11, 660-669. doi: 10.1017/S1138741600004662

$\mathrm{Hu}$, L., y Bentler, P. (1999). Cutoff criteria for fit indexes in covariance structure analysis: Conventional criteria versus new alternatives. Structural Equation Modeling, 6(1), 1-55. doi: 10.1080/10705519909540118.

Jackson, S., y Csíkszentmihályi, M. (2002). Fluir en el Deporte. Badalona: Paidotribo.

Jackson, S., y Eklund, R.C. (2002). Assessing flow in physical activity: The Flow State Scale-2 and Dispositional Flow Scale-2. Journal of Sport and Exercise Psychology, 24, 133-150.

Jackson, S., y Eklund, R.C. (2004). Relationships between quality of experience and participation in diverse performance settings. Australian Journal of Psychology, 56(Suppl), 193.

Jackson, S., y Marsh, H. (1996). Development and validation of a scale to measure optimal experience: The Flow State Scale. Journal of Sport and Exercise Psychology, 18, 17-35.

Landhäuber, A., y Keller, J. (2012). Flow and Its Affective, Cognitive, and Performance-Related Consequences. En S. Engeser (ed.), Advances in Flow Research (pp. 65-85). London: Springer. doi: 10.1007/978-1-4614-2359-1_4

Mesurado, B. (2009). Actividad estructurada vs. actividad desestructurada, realizadas en solitario $v s$. en compañía de otros y la experiencia óptima. Anales de Psicología, 25(2), 308-315. Recuperado de: http://revistas.um.es/analesps/article/view/87711/84501

Moneta, G. (2012). On the Measurement and Conceptualization of Flow. En S. Engeser (Ed.), Advances in Flow Research (pp. 23-50). London: Springer. doi: 10.1007/978-1-46142359-1_2

Páez, D., Rimé, B., Basabe, N., Wlodarczyk, A., y Zumeta, L. (2015). Psychosocial Effects of Perceived Emotional Synchrony in Collective Gatherings. Journal of Personality and Social Psychology, 108(5), 711-729. doi.org/10.1037/pspi0000014.

Peifer, C. (2012). Psychophysiological correlates of Flow-experience. En S. Engeser (ed.), Advances in Flow Research (pp. 139-164). London: Springer. doi: 10.1007/978-1-4614-2359-1_8

Preacher, K., y Hayes, A. (2008). Asymptotic and resampling strategies for assessing and comparing indirect effects in multiple mediator models. Behavior Research Methods, 40, 879-891. doi: 10.3758/BF03206553 
Salanova, M., Rodríguez-Sánchez, A.M., Schaufeli, W.B., y Cifre, E. (2014). Flowing together: a longitudinal study of collective efficacy and collective flow among workgroups. Journal of Psychology, 148, 435-455. doi: 10.1080/00223980.2013.806290

Sampedro, P. (2015). Efecto del ejercicio aeróbico y la estimulación ambiental sobre la reducción de los niveles de ansiedad en el envejecimiento. European Journal of Investigation in Health, Psychology and Education, 5(2), 197-208. doi: 10.1989/ejihpe.v5i2.112

Schiepe-Tiska, A., y Engeser, S. (2012). Flow in non-achievement situations. En S. Engeser (Ed.), Advances in flow research (pp. 87-107). New York: Springer. doi: 10.1007/978-1-46142359-1_5

Vázquez, C., y Hervas, G. (2013). Addressing current challenges in cross-cultural measurement of well-being: The Pemberton Happiness Index. En A. Delle Fave y H.H. Koop (Eds), Anthology on Cross Cultural Advancements in Positive Psychology (pp. 31-49). New York: Springer-Verlag. doi: 10.1007/978-94-007-4611-4_3

Walker, C. (2010). Experiencing flow: Is doing it together better than doing it alone? Journal of Positive Psychology, 5(1), 3-11. doi: 10.1080/17439760903271116

Wiltermuth, S.S., y Heat, C. (2009). Synchrony and Cooperation. Psychological Science, 20, 1-5. doi: 10.1111/j.1467-9280.2008.02253.x

Zumeta, L.N., Oriol, X., Telletxea, S., Amutio, A., y Basabe, N. (2016). Collective Efficacy in Sports and Physical Activities: Perceived Emotional Synchrony and Shared Flow. Frontiers in Psychology. Movement Science and Sport Psychology, 1960. doi: 10.3389/fpsyg.2015.01960.

Recibido: 18 de febrero de 2016

Recepción Modificaciones: 25 de mayo de 2016

Aceptado: 27 de mayo de 2016 